\title{
Polarité planaire chez les Mammifères : similitudes et divergences avec la Drosophile
}

\author{
par Mireille Montcouquiol \\ Équipe Avenir 2, INSERM, Institut Francois Magendie, Université Bordeaux II, 146, rue Léo-Saignat, \\ 33077 Bordeaux Cedex, France. Tél. : (33) 5575737 62. Fax : (33) 557573752. \\ E-mail : montcouquiol@bordeaux.inserm.fr \\ Reçu le 26 juin 2006
}

\section{RÉSUMÉ}

Les gènes de la polarité planaire (PP) furent initialement découverts chez les Invertébrés (Drosophila Melanogaster) pour leur rôle dans l'orientation uniforme de structure (cils, groupes de cellules) dans le plan de l'épithélium. Au cours de ces cinq dernières années, de nombreux travaux ont montré que chez les Vertébrés, et en particulier les Mammifères, certains homologues de ces gènes sont importants dans des mécanismes développementaux aussi divers que la fermeture de la gouttière neurale, la maladie polykystique du rein, les fonctions de l'oreille interne (audition, équilibre) ou encore la maladie de BardetBiedl. Ces processus font appel à un groupe de gènes dont l'implication dans des mécanismes de PP est conservée au cours de l'évolution pour certains, ou uniquement présente chez les Mammifères pour d'autres. En 2003, l'identification de la cochlée comme modèle d'étude de la PP chez les Vertébrés a ouvert la voie à l'identification des gènes importants chez les Mammifères. Les protéines codées par ces gènes sont des protéines de surface et des protéines de la signalisation intracellulaire dont les mécanismes d'action restent encore énigmatiques. Il semble de plus en plus évident que notre compréhension des mécanismes de la PP chez les Mammifères passe par l'analyse de gènes homologues à ceux de la Drosophile, mais également par l'identification de gènes spécifiques aux Mammifères.

\section{SUMMARY Planar polarity in mammals: similarity and divergence with Drosophila Melanosgaster}

Planar cell polarity (PCP) genes were originally identified in invertebrates (Drosophila Melanogaster) for their role in the uniform orientation of a structure within the plane of the epithelium (hair, group of cells). During the last five years, numerous studies have shown that vertebrate, but more importantly, mammalian homologues of some of these genes are involved in various developmental processes such as neural tube closure, polycystic kidney disease, inner ear functions (hearing, balance) or Bardet Biedl syndrome. These processes rely on a set of genes whose PCP function is conserved in mammals and Drosophila Melanogaster for some, or only present in mammals for others. In 2003, the inner ear was identified as a model to study PP in mammals and allowed the identification of the first important genes. These genes encode a variety of cell surface molecules as well as intracellular adapters whose molecular mechanisms are still poorly understood. It is clear that the identification of the PP pathways in mammals will come from a comparison with the genes in Drosophila, but also from the identification of genes specific to mammals.
La voie de signalisation des Wnt fut initialement identifiée comme la voie qui stabilisait la $\beta$-caténine, et appelée voie canonique (Logan \& Nusse, 2004 ; Nusse, 2005). Des travaux plus récents ont montré l'existence d'au moins deux autres voies de signalisation par les Wnt, et ces nouvelles cascades furent donc nommées non canoniques (Strutt, 2003; Veeman et al., 2003). La découverte de ces voies de signalisation a également permis de mettre à jour de nouvelles fonctions régulées par les Wnt et/ou leurs récepteurs, les Frizzled. En particulier, la régulation de la polarisation cellulaire est une des fonctions majeures contrôlées par ces cascades non cano- niques. En fait, la notion de polarisation s'est considérablement élargie ces dernières années et comprend maintenant les mouvements cellulaires coordonnés, la migration cellulaire, et l'orientation uniforme dans le plan d'un épithélium.

\section{VOIES CANONIQUE ET NON-CANONIQUES}

Dix-neuf gènes Wnt ont été identifiés chez les Vertébrés contre seulement sept wingless (wg) chez la Drosophile. L'activation de la voie de signalisation cano- 
nique des Wnt conduit à l'inhibition de la Glycogen

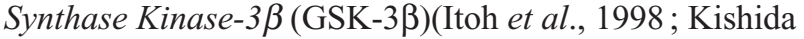
et al., 1999; Peters et al., 1999; Smalley et al., 1999), résultant en une stabilisation de la $\beta$-caténine (Rubinfeld et al., 1996; Yost et al., 1996; Yamamoto et al., 1999). Lorsque la $\beta$-caténine est accumulée en quantité suffisante dans le cytoplasme d'une cellule, elle se transloque dans le noyau, où elle peut activer la transcription de gènes en formant un complexe avec un membre de la famille des facteurs transcriptionnels TCF/Lef (Behrens et al., 1996; Molenaar et al., 1996; Hart et al., 1999).

Pour activer la voie canonique, les Wnt doivent se lier aux membres de la famille des récepteurs Frizzled $(\mathrm{Fz})$, qui comprend au moins dix membres chez la souris (Huang \& Klein, 2004). Ces récepteurs se présentent sous la forme de protéines à sept domaines transmembranaires, dont l'activation peut être modulée par des co-récepteurs tels les LRP ( $L D L$ Receptor associated Protein). La liaison des Wnt aux Fz entraîne la phosphorylation de Disheveled, une protéine cytosolique, qui en retours inhibe GSK-3 $\beta$. Il existe trois gènes disheveled $(D v l)$ chez les Mammifères, et un seul $(d s h)$ chez la mouche Drosophila Melanogaster (Perrimon \& Mahowald, 1987; Sussman et al., 1994; Klingensmith et al., 1996; Tsang et al., 1996).

Des manipulations chez le Xénope embryonnaire ont montré que l'activation de la voie canonique par certains Wnt (Wnt1 ou Wnt8 par exemple) conduit à une duplication de l'axe antéropostérieur (Glinka et al., 1997; Sokol et al., 1991). L'identification d'une autre voie de signalisation est venue de l'observation que certains Wnt (Wnt4, Wnt5a et Wnt11) entraînent des problèmes de gastrulation, mais pas de duplication de l'axe (Du et al., 1995; Moon et al., 1993; Tada \& Smith, 2000). Il est maintenant clair que l'activation de la voie canonique influencera le destin cellulaire et la duplication de l'axe antéropostérieur, alors que la voie non-canonique influencera la gastrulation. Lors de l'activation de la voie non-canonique, les Wnt se lient aussi aux récepteurs Fz, et Dvl est également activé, mais cette activation n'entraîne pas la stabilisation de la $\beta$-caténine. Encore plus surprenant, il semble que les Wnt ne soient pas requis dans certains contextes, bien que les $\mathrm{Fz}$ et Dvl, eux, soient toujours nécessaires.

$\mathrm{Au}$ moins deux de ces voies non-canoniques ont été identifiées : la voie calcium/PKC et la voie de la polarité planaire. La voie calcium/PKC met en jeu certain Wnt (Wnt5a ou Wnt11) dont la liaison aux récepteurs Fz ne conduit pas à une stabilisation de la $\beta$-caténine, mais à une augmentation calcique intracellulaire dans l'ovocyte de Xenope (Slusarski et al., 1997; Kuhl et al., 2000). Parfois cette augmentation de calcium est accompagnée de l'activation de la calcium/calmodulin-dependent kinase (CAMKII) et de la protéine kinase C (PKC) (Sheldahl et al., 1999; Kuhl et al., 2000). La voie de la polarité planaire (PP), elle, utilise Fz et Dvl, ainsi qu'un ensemble de molécules membranaires et cytosoliques, mais en l'absence de Wnt (Mlodzik, 2002; Fanto \& McNeill, 2004).

\section{POLARITÉ PLANAIRE (PP)}

Certaines cellules épithéliales possèdent une polarité dont l'axe est perpendiculaire à l'axe apico-basal, et qui est appelée polarité planaire. Cette définition simple provient initialement de l'observation des poils qui couvrent la cuticule des insectes, et qui sont tous orientés de façon uniforme dans l'axe du corps. La polarité planaire est en fait présente sous de nombreux aspects dans notre vie courante et on la retrouve par exemple dans l'orientation uniforme des écailles des Poissons, des plumes des Oiseaux ou de la touffe ciliaire des cellules auditives dans l'oreille interne des Mammifères. En fait, la mouche Drosophila Melanogaster a été un outil majeur dans la découverte des gènes de la polarité planaire, grâce à l'étude principalement de ses ailes et de ses yeux, mais aussi de son abdomen ou de ses pattes. Parmi tous ces tissus, l'aile de Drosophile est le système d'analyse de la PP le plus simple. Les cellules de l'aile de Drosophile produisent chacune un poil épidermique en position apicale. Ce poil, riche en tubuline, sera toujours positionné dans la partie distale de la cellule, et orienté dans l'axe de l'épithélium - l'aile - de façon proximo-distale chez l'animal sauvage (Fig. 1A). Tous les poils couvrant l'aile sont donc orientés de manière uniforme, et il en résulte une visualisation très simple de la PP, mais également de toute perturbation de cette PP. Cette particularité a permis l'identification d'un groupe de gènes dont la mutation affecte cette orientation uniforme. Ce groupe, appelé "core genes" regroupe six gènes dont la mutation affecte fortement la PP chez la Drosophile, quel que soit le tissu étudié. Il regroupe les gènes frizzled $(f z)$, dishevelled (dsh), prickle (pk), strabismus/van Gogh (stbm/vang), flamingo ( $f m i)$ et diego.

\section{LOCALISATION ASYMÉTRIQUE DES PROTÉINES DE LA PP}

Une des particularités des "core genes" est que la mutation de chacun de ces gènes affecte également la localisation des autres protéines du groupe. En fait, l'établissement de l'orientation proximo-distale du poil épidermique reflète la distribution proximo-distale des protéines issues de la traduction des six "core genes". Dans l'aile de Drosophile, quelques heures avant la formation du poil, les protéines de la PP sont recrutées au cortex membranaire en position apico-latérale, au niveau de la zonula adherens des cellules. Leur distribution initiale est homogène, mais très vite, une redistribution proximo-distale s'opère, confinant certaines protéines du côté distal et d'autres du côté proximal de la cellule (Fig. 1A). Il en résulte que le poil se développera toujours du côté distal de la cellule, qui correspond à l'accumulation de fz et dsh, mais à l'opposé de stbm/vang et pk (Strutt, 2002). La mutation de chacun des "core genes" conduira à la perte de cette asymétrie et à la perturbation de l'orientation du poil, soulignant les fortes interactions liant les protéines de ce complexe. 


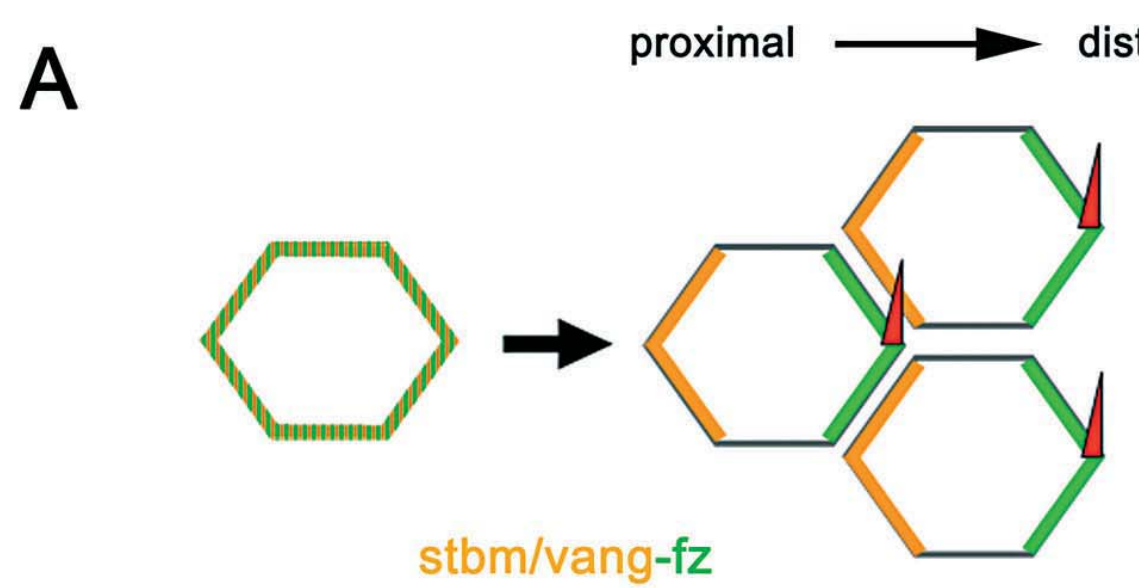

Modifié d'après Strutt D. (2003)
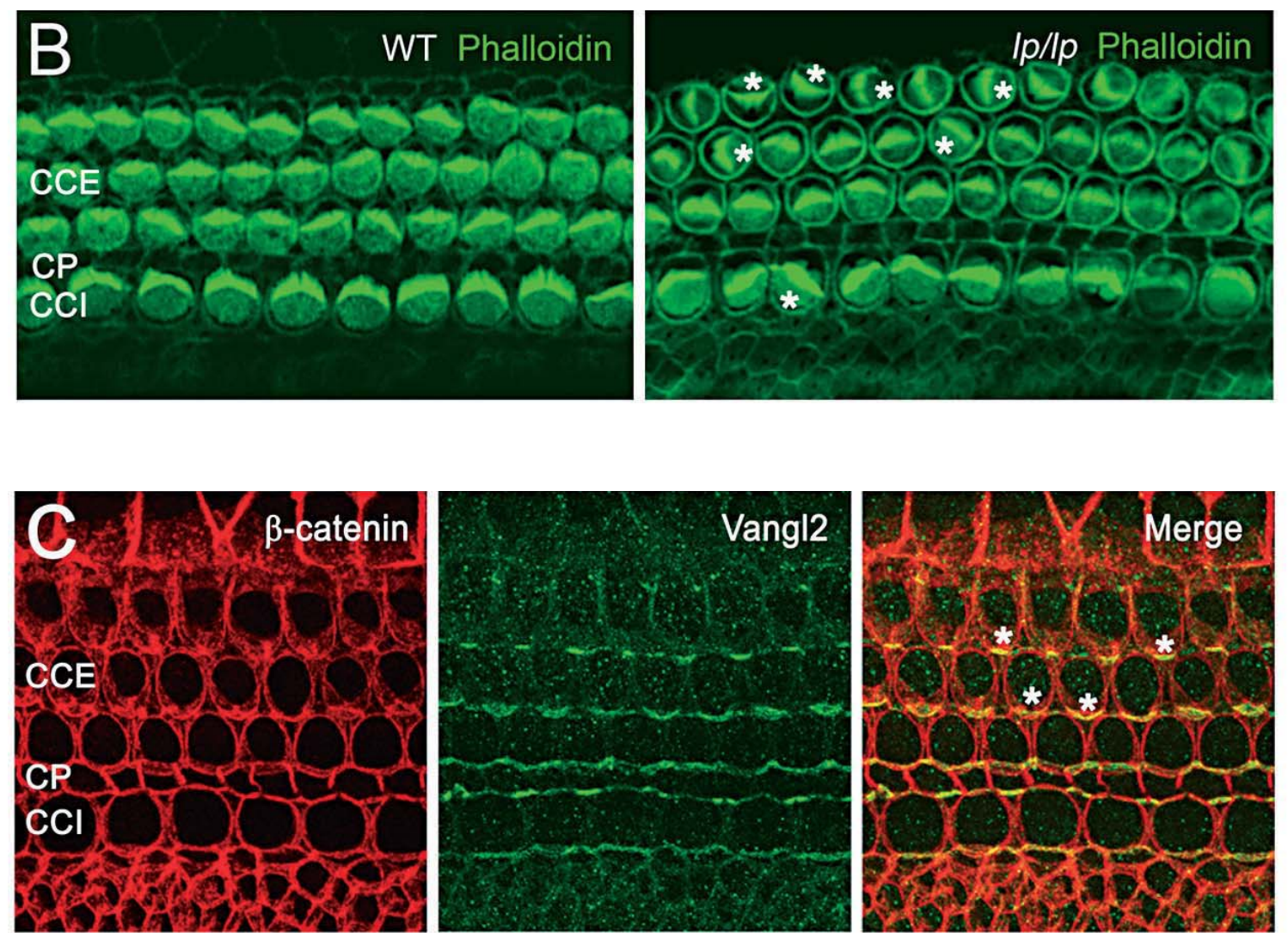

Fig. 1. - A. Représentation schématique de la distribution de deux protéines de la PP dans les cellules d'aile de Drosophile : vang (orange) et fz (vert). Quelques heures avant l'apparition des poils épidermiques dans la partie apicale des cellules, vang et fz sont recrutées dans la zonula adherens, de façon homogène. Juste avant l'apparition du poil (représenté en rouge pour faciliter la compréhension), une redistribution des protéines a lieu, limitant vang à la partie proximale de la cellule, et fz à la partie distale, où se développera le poil (schéma modifié d'après Strut, 2003). B. Vues de surface d'une cochlée de souris (WT) et de looptail homozygote (lp/lp) marquées à la phalloïdine (vert) afin d'identifier les touffes ciliaires riches en actine. Chez l'animal sauvage, on retrouve l'organisation rigoureuse de l'organe de Corti, avec une rangée de cellules ciliées internes (CCI), séparées par les cellules piliers (CP) par trois rangées de cellules ciliées externes (CCE). Le marquage à la phalloïdine permet de visualiser l'orientation uniforme des touffes ciliaires dans l'épithélium sensoriel. Par comparaison l'orientation des touffes ciliaires est perturbée chez l'animal $l p / l p$, portant une mutation sur Vangl2, et l'on observe plusieurs touffes ciliaires qui ne sont pas orientées uniformément (astérisques). C. Vues de surface d'un épithélium sensoriel de cochlée de rat néonatal illustrant la distribution asymétrique de Vang12 (en vert). Le marquage à la $\beta$-caténine permet de visualiser les membranes des cellules (rouge), alors que le marquage pour Vangl2 montre clairement une localisation proximo-distale de la protéine (vert). L'accumulation de Vangl2 est particulièrement prononcée à la jonction des CCE et des cellules de soutien qui les contactent (astérisques), mais également au niveau des CP. 


\section{ÉLARGISSEMENT DE LA NOTION DE POLARITÉ PLANAIRE ET IMPLICATION DANS DES MALADIES}

Une accumulation de données ces cinq dernières années suggère que les protéines de la PP ne sont pas impliquées dans une cascade spécifique, mais plus probablement dans différentes cascades, et que cette diversité permet de contrôler divers mécanismes développementaux chez les Invertébrés et les Vertébrés.

Par exemple, chez la Drosophile, un mécanisme dépendant de $s t b m / v a n g$ régule le lignage des organes sensoriels du thorax dorsal en contrôlant la division asymétrique du précurseur du lignage (Bellaiche et al., 2001, 2004; Veeman et al., 2003). Ce mécanisme représente une réelle $\mathrm{PP}$, dont la cascade met en jeu seulement certaines des protéines des "core genes" (comme $f z$ et $\mathrm{stbm} /$ vang), et dont la voie de signalisation est différente de celle mise en œuvre lors de la PP des poils épidermiques de l'aile de Drosophile. D'autres travaux chez le Poisson zèbre (Danio Rerio) et la Grenouille (Xenopus Laevis) montrent que les homologues des gènes $f z, d s h$, $\mathrm{pk}, \mathrm{stbm} / \mathrm{vg}$, fmi ou diego agissent de manière concertée pour contrôler différents mouvements cellulaires au cours de la gastrulation (et en particulier l'extension convergente) et de la neurulation (fermeture du tube neural) (Keller, 2002, 2005). La perturbation de cette dernière entraîne des problèmes sévères de la fermeture de la gouttière neurale, qui affecte approximativement un enfant sur mille (Copp, 2003). Les cellules semblent donc communiquer entre elles pour adopter une même orientation vectorielle dans le plan de l'épithélium et/ou répondent de manière convergente à un signal de polarisation agissant sur l'ensemble du tissu, en utilisant les homologues - ou en tout cas une partie - des "core genes" de la Drosophile. Plus récemment, deux études ont encore élargi notre vision de la fonction de certains gènes de la polarité planaire chez les Mammifères en les impliquant dans la maladie polykystique des reins (PKD) (Fischer et al., 2006) et le syndrome de Bardet-Biedl (Ross et al., 2005). Ces deux études soulignent la diversité des mécanismes contrôlés par les protéines de la polarité planaire et leurs impacts sur des maladies identifiées chez l'Homme, mais dont on ne connaît pas ou peu les mécanismes moléculaires. Il est important de souligner que tous ces divers mécanismes contrôlés par les protéines de la PP ont un dénominateur commun : la modification du cytosquelette des cellules concernées.

\section{NOUVEAUX GĖNES SPÉCIFIQUES DE LA PP DES VERTÉBRÉS}

Au moins deux nouveaux gènes de la PP ont été isolés chez les Vertébrés sans qu'on leur connaisse de rôle ou d'existence chez les Invertébrés : la neurotrophinreceptor-related protein, $N R H 1$, et la protein tyrosine kinase 7 (PTK7/CCK-4). Le gène NRHI fut isolé initialement d'un crible visant à analyser les gènes exprimés dans le neuroectoderme de Xénope (Sasai et al., 2004). La surexpression de ce gène chez le même animal entraîne une perturbation des mouvements d'extension convergente. Lors d'un autre crible visant à trouver des protéines transmembranaires importantes au cours du développement du système nerveux chez la Souris, la protéine PTK7 fut identifiée. Une mutation dans le gène codant pour PTK7 conduit à des perturbations de l'extension convergente, de la fermeture du tube neural, mais également de l'orientation des touffes ciliaires des cellules de l'oreille interne (Lu et al., 2004). De plus, la démonstration d'une interaction génétique entre Vangl2 (l'orthologue de $s t b m / v g$ ) et PTK7 montre clairement une implication de ce gène dans la/les voie(s) de signalisation de la PP chez les Mammifères.

L'identification de nouveaux gènes de la PP chez les Vertébrés suggère un degré de complexité additionnel nécessaire à la régulation fine et précise de multiples mécanismes. Il ne fait aucun doute que d'autres gènes et mécanismes restent à découvrir chez les Vertébrés, et plus particulièrement chez les Mammifères.

\section{POLARITÉ PLANAIRE CHEZ LES MAMMIFÈRES : L'OREILLE INTERNE}

L'épithélium sensoriel de l'oreille interne des Mammifères, l'organe de Corti, est une des structures les plus finement organisées chez les Vertébrés (Fig. 1B). Il est composé de six types cellulaires uniques organisés en une mosaïque : deux types de cellules mécano-sensorielles et quatre types de cellules de soutien, alignées sur toute la longueur du canal cochléaire. Chaque cellule sensorielle possède une touffe de stéréocils ou touffe ciliaire à son apex, indispensable aux fonctions d'audition de la cochlée et d'équilibre du système vestibulaire. Les cellules sensorielles ne sont sensibles à des déflections que dans un plan unique de l'espace, et l'épithélium a donc dû développer une polarité uniforme de façon à ce que toutes les cellules soient orientées dans le même plan, grâce à la PP (Hudspeth, 1997; Eaton, 1997, 2003). La perturbation de l'orientation des touffes ciliaires entraîne donc une diminution ou perte de fonction auditive et de l'équilibre (Yoshida \& Liberman, 1999).

En 2003, nous avons décrit l'orientation des touffes ciliaires de cochlées de souris mutantes pour le gène Van Gogh like-2 (Vangl2), l'orthologue de stbm/vang, et pour le gène Scribblel, l'orthologue de scribble chez la Drosophile (Montcouquiol et al., 2003). Nous avons montré que les touffes ciliaires des cellules sensorielles chez les homozygotes looptail ( $l p / l p$; mutant pour Vangl2) et circletail ( $c r c / c r c$; mutant pour scribl) étaient fortement perturbées par rapport à celles d'animaux contrôles (Fig. 1B). Ces résultats ont permis d'identifier vangl2 et scribl comme les deux premiers gènes de la polarité planaire chez les Mammifères. Comme cela a été discuté plus haut, une mutation de vang chez la Drosophile conduit à une désorganisation de la PP dans des structures épithéliales telles que l'aile ou l'œil. Le phénotype 
de l'oreille interne des mutants pour Vangl2, suggère donc une conservation de la fonction du gène entre la Drosophile et les Mammifères. Le gène scribble par contre n'a pas de rôle connu dans la PP chez la Drosophile, et a été en fait identifié pour son rôle lors de l'établissement de la polarité apico-basale des cellules épithéliales (Bilder \& Perrimon, 2000). Il reste cependant possible que la sévérité du phénotype chez la mouche mutante pour scribble empêche l'observation d'un rôle lors de l'établissement de la PP. Il est en effet raisonnable d'imaginer que polarité apico-basale et PP sont des processus sinon concomitants, en tout cas très proches dans le temps. Deux études récentes suggèrent même des liens étroits entre molécules de la polarité apicobasale et de la PP (Djane et al., 2005 ; Dollar et al, 2005).

En plus d'identifier Vangl2 et Scribl comme gènes de la PP, notre étude a également permis d'imposer la cochlée comme l'un des meilleurs systèmes d'étude de la PP chez les Mammifères. En effet, sur les sept gènes identifiés à ce jour chez les Mammifères (vangl2, scrib1, celsrl, PTK7, $d v l 1-2, f z 3-6)$, tous l'ont été sur la base du phénotype de l'oreille interne des mutants (Montcouquiol et al., 2003; Curtins et al., 2003; Lu et al., 2004 ; Guo et al., 2004; Wang et al., 2005, 2006).

\section{ASYMÉTRIE DES PROTÉINES DE LA PP CHEZ LES MAMMIFÈRES}

Comme on l'a vu précédemment, l'asymétrie des protéines de la PP chez la Drosophile est une caractéristique importante de ces molécules, bien que l'on ne connaisse pas les mécanismes moléculaires mis en jeu lors de cette asymétrie, ni vraiment ce qu'elle détermine.

Dans un effort pour comprendre si les Mammifères possèdent de telles propriétés, nous avons développé un anticorps pour l'une d'entre elles, la protéine transmembranaire Vang12. Nos résultats montrent que tout comme dans l'aile ou l'œil de Drosophile, Vang12 est localisée de façon asymétrique - proximo-distale - dans la cochlée de Mammifère (Fig. 1C). Plus spécifiquement, la protéine semble s'accumuler dans la partie proximale des cellules sensorielles, c'est-à-dire à l'opposé de la touffe ciliaire, tout comme vang est localisé en position proximale des cellules de l'aile de Drosophile, à l'opposé du poil épidermique. En fait, la localisation asymétrique de Vang12 n'est probablement pas aussi simple qu'une localisation proximale dans les cellules ciliées, mais semble mettre en jeu les cellules de soutien qui les touchent et les séparent (Fig. 1C) (Wang et al., 2006; Montcouquiol et al., 2006). La multiplication des types cellulaires formant l'épithélium sensoriel de cochlée par rapport au type cellulaire unique de l'aile de Drosophile a probablement requis une complexification des mécanismes mis en jeu lors de la ségrégation des protéines de la PP.

Chez la Drosophile, une mutation de flamingo ( $\mathrm{fmi}$ ) entraîne non seulement une perte d'asymétrie des autres protéines, mais perturbe également le recrutement de ces protéines à la membrane (Strutt, 2001). De plus, nous savons qu'une mutation sur le gène celsrl (l'un des trois orthologues de fmi chez le Mammifère) conduit à des problèmes d'orientation des touffes ciliaires dans l'oreille de mutant crsh (Curtin et al., 2003). Une immunocytochimie de cochlée de crsh/crsh mutant montre que la mutation de la cadhérine atypique celsrl conduit également à une diminution dramatique du recrutement de Vang12 à la membrane des cellules de l'épithélium sensoriel. Ces deux exemples illustrent la conservation des mécanismes et des fonctions de la PP entre Vertébrés et Invertébrés.

Pourtant, il existe également des différences importantes avec les Invertébrés. Le rôle de la protéine Scrib1 chez les Mammifères, par exemple, est très étonnant. Après avoir confirmé l'interaction génétique de Vangl2 avec Scrib1 en démontrant une interaction protéique des deux molécules, nous avons montré que la protéine cytosolique Scrib1 est requise pour la localisation asymétrique de Vang12 dans l'oreille de Mammifère, alors qu'elle n'est pourtant pas localisée asymétriquement ellemême (Montcouquiol et al., 2006).

Un autre résultat inattendu concerne les récepteurs $\mathrm{Fz}$ et fut décrit initialement par le laboratoire de Jeremy Nathans. Wang et al. (2006) ont décrit très récemment un double mutant pour $F z 3$ et $F z 6$, deux des dix récepteurs $\mathrm{Fz}$ chez la souris, et ont montré que les deux protéines étaient impliquées dans la polarité planaire de l'oreille de Mammifêre (Wang et al., 2006). Leur étude décrit également une localisation asymétrique des deux récepteurs sur la partie proximale des cellules ciliées, résultat que nous avons confirmé pour Fz3. Leurs résultats et les nôtres suggèrent donc une co-localisation des deux $\mathrm{Fz}$ avec Vang12. En fait, nous avons réussi à immunoprécipiter Vang12 et Fz3, suggérant ainsi une interaction directe entre les deux protéines. Cette interaction pourrait en fait être nécessaire au recrutement ou à la stabilisation des protéines à la membrane, car en l'absence de Vang12, Fz3 n'est plus présent à la membrane, et viceversa. Ces résultats sont particulièrement surprenants car ils sont très différents des mécanismes évoqués jusqu'à présent chez la Drosophile, où classiquement stbm/vang et Fz sont exprimés soit sur des côtés opposés (proximodistal) d'une même cellule (aile de Drosophile), soit sur les membranes en vis-à-vis de deux cellules voisines (œil de Drosophile). Il est bien évident que nous n'avons encore qu'une vision partielle des éléments et mécanismes régissant la $\mathrm{PP}$ chez les Mammifères, et que d'autres études seront nécessaires à la compréhension de cette voie de signalisation, dont nous n'avons encore qu'effleuré la complexité. Ces résultats soulignent en fait l'importance d'une analyse spécifique des protéines chez les Mammifères, de façon à identifier les différences entre les cascades moléculaires de la PP chez les Invertébrés et les Vertébrés.

\section{CONCLUSION}

Des études récentes ont souligné les similitudes et les divergences existant entre la PP chez les Invertébrés, et chez les Vertébrés, et plus particulièrement les Mammi- 
fères. Ces études commencent à peine à nous donner des informations sur les molécules et les cascades moléculaires impliquées lors de la mise en place de la PP. En particulier, les molécules en aval des molécules transmembranaires de la PP et impliquées dans le remodelage du cytosquelette nous sont encore inconnues. Étant donné la diversité des mécanismes dans lesquels les molécules de la PP sont impliquées, il semble clair que l'élucidation de tels mécanismes sera primordiale pour nous éclairer sur les problèmes développementaux que leurs perturbations entrainent chez les Mammifères.

\section{BIBLIOGRAPHIE}

Behrens J., von Kries J. P., Kuhl M., Bruhn L., Wedlich D., Grosschedl R. \& Birchmeier W., Functional interaction of $\beta$ catenin with the transcription factor LEF-1. Nature, 1996, 382, 638-642.

Bellaiche Y., Radovic A., Woods D. F., Hough C. D., Parmentier M. L. , O'Kane C. J., Bryant P. J. \& Schweisguth F., The partner of inscuteable/discs-large complex is required to establish planar polarity during asymmetric cell division in Drosophila. Cell, 2001, 106, 355-366.

Bellaiche Y., Beaudoin-Massiani O., Stuttem I. \& Schweisguth F., The planar cell polarity protein Strabismus promotes Pins anterior localization during asymmetric division of sensory organ precursor cells in Drosophila. Development, 2004, $131,469-478$.

Bilder D. \& Perrimon N. Localization of apical epithelial determinants by the basolateral PDZ protein Scribble. Nature, 2000, 403, 676-680.

Copp A. J., Greene N. D. \& Murdoch J. N., The genetic basis of mammalian neurulation. Nat. Rev. Genet., 2003, 4, 784-493.

Curtin J. A. et al., Mutation of Celsr1 disrupts planar polarity of inner ear hair cells and causes severe neural tube defects in the mouse. Curr. Biol., 2003, 13, 1129-1133.

Djane A., Yogev S. \& Mlodzik M., The apical determinants aPKC and dPatj regulate Frizzled-dependent planar cell polarity in the Drosophila eye. Cell, 2005, 121, 621-631.

Dollar G. L., Weber U., Mlodzik M. \& Sokol S. Y., Regulation of Lethal giant larvae by Dishevelled. Nature, 2005, 437, 13761380 .

Du S. J., Purcell S. M., Christian J. L., McGrew L. L. \& Moon R. T., Identification of distinct classes and functional domains of Wnts through expression of wild-type and chimeric proteins in Xenopus embryos. Mol. Cell. Biol., 1995, $15,2625-2634$

Eaton S., Cell biology of planar polarity transmission in the Drosophila wing. Mech. Dev., 2003, 120, 1257-1264.

Eaton S., Planar polarization of Drosophila and vertebrate epithelia. Curr. Opin. Cell., Biol. 1997, 9, 860-866.

Fanto M. \& McNeill H., Planar polarity from flies to vertebrates. J. Cell. Sci., 2004, 117, 527-533.

Fischer E., Legue E., Doyen A., Nato F., Nicolas J. F., Torres V., Yaniv M. \& Pontoglio M., Defective planar cell polarity in polycystic kidney disease. Nat. Genet., 2006, 38, 21-23.

Glinka A., Wu W., Onichtchouk D., Blumenstock C. \& Niehrs C., Head induction by simultaneous repression of Bmp and Wnt signalling in Xenopus. Nature, 1997, 389, 517-519

Guo N., Hawkins C. \& Nathans J., Frizzled6 controls hair patterning in mice. Proc. Natl. Acad. Sci. USA, 2004, 101, 92779281.

Hart M., Concordet J. P., Lassot I., Albert I., del los Santos R., Durand H., Perret C., Rubinfeld B., Margottin F., Benarous R. \& Polakis P., The F-box protein $\beta$ - $\operatorname{TrCP}$ associates with phosphorylated $\beta$-catenin and regulates its activity in the cell. Curr. Biol., 1999, 9, 207-210.

Huang H. C. \& Klein P. S., The Frizzled family: receptors for multiple signal transduction pathways. Genome Biol., 2004, 5, 234.

Hudspeth A. J., How hearing happens. Neuron, 1997, 19, 947950.

Itoh K., Krupnik V. E. \& Sokol S. Y., Axis determination in Xenopus involves biochemical interactions of axin, glycogen synthase kinase 3 and $\beta$-catenin. Curr. Biol., 1998, 8, 591-594.

Keller R., Shaping the vertebrate body plan by polarized embryonic cell movements. Science, 2002, 298, 1950-1954

Keller R., Cell migration during gastrulation. Curr. Opin. Cell. Biol., 2005, 17, 533-541.

Kishida S., Yamamoto H., Hino S., Ikeda S., Kishida M. \& Kikuchi A. DIX domains of Dvl and axin are necessary for protein interactions and their ability to regulate $\beta$-catenin stability. Mol. Cell. Biol., 1999, 19, 4414-4422.

Klingensmith J., Nusse R. \& Perrimon N., The Drosophila segment polarity gene dishevelled encodes a novel protein required for response to the wingless signal. Genes Dev., 1994, 8, 118-130.

Kuhl M., Sheldahl L. C., Park M., Miller J. R. \& Moon R. T., The $\mathrm{Wnt} / \mathrm{Ca}^{+}$pathway: a new vertebrate Wnt signaling pathway takes shape. Trends Genet., 2000, 16, 279-283.

Logan C. Y. \& Nusse R., The Wnt signaling pathway in development and disease. Annu. Rev. Cell. Dev. Biol., 2004, 20, 781-710.

Lu X., Borchers A. G., Jolicoeur C., Rayburn H., Baker J. C. \& Tessier-Lavigne M., PTK7/CCK-4 is a novel regulator of planar cell polarity in vertebrates. Nature, 2004, 430, 93-98.

Mlodzik M., Planar cell polarization: do the same mechanisms regulate Drosophila tissue polarity and vertebrate gastrulation? Trends Genet., 2002, 18, 564-571.

Molenaar M., van de Wetering M., Oosterwegel M., PetersonMaduro J., Godsave S., Korinek V., Roose J., Destree O. \& Clevers H., XTcf-3 transcription factor mediates $\beta$-catenininduced axis formation in Xenopus embryos. Cell, 1996, 86, 391-399.

Montcouquiol M., Rachel R. A., Lanford P. J., Copeland N. G., Jenkins N. A. \& Kelley M. W., Identification of Vangl2 and Scrb1 as planar polarity genes in mammals. Nature, 2003, 423, 173-177.

Moon R. T., Campbell R. M., Christian J. L., McGrew L. L., Shih J. \& Fraser S., Xwnt-5A: a maternal Wnt that affects morphogenetic movements after overexpression in embryos of Xenopus laevis. Development, 1993, 119, 97-111.

Nusse R. Wnt signaling in disease and in development. Cell. Res. $2005,15,28-32$.

Perrimon N. \& Mahowald A. P., Multiple functions of segment polarity genes in Drosophila. Dev. Biol., 1987, 119, 587 600.

Peters J. M., McKay R. M., McKay J. P. \& Graff J. M., Casein kinase I transduces Wnt signals. Nature, 1999, 401, 345350.

Ross A. J., May-Simera H., Eichers E. R., Kai M., Hill J., Jagger D. J., Leitch C. C., Chapple J. P., Munro P. M., Fisher S., Tan P. L., Phillips H. M., Leroux M. R., Henderson D. J., Murdoch J. N., Copp A. J., Eliot M. M., Lupski J. R., Kemp D. T., Dollfus H., Tada M., Katsanis N., Forge A. \& Beales P. L., Disruption of Bardet-Biedl syndrome ciliary proteins perturbs planar cell polarity in vertebrates. Nat. Genet., 2005, 37, 1135-1140.

Rubinfeld B., Albert I., Porfiri E., Fiol C., Munemitsu S. \& Polakis P., Binding of GSK3 $\beta$ to the APC- $\beta$-catenin complex and regulation of complex assembly. Science, 1996, 272, 1023-1026.

Sasai N., Nakazawa Y., Haraguchi T. \& Sasai Y., The neurotrophin-receptor-related protein NRH1 is essential for conver- 
gent extension movements. Nature Cell. Biol., 2004, 6, 741748.

Sheldahl L. C., Park M., Malbon C. C. \& Moon R. T., Protein kinase $\mathrm{C}$ is differentially stimulated by Wnt and Frizzled homologs in a G-protein-dependent manner. Curr. Biol., 1999, 9, 695-698.

Slusarski D. C., Yang-Snyder J., Busa W. B. \& Moon R. T., Modulation of embryonic intracellular $\mathrm{Ca}^{+}$signaling by Wnt5A. Dev. Biol., 1997, 182, 114-120.

Smalley M. J., Sara E., Paterson H., Naylor S., Cook D., Jayatilake H., Fryer L. G., Hutchinson L., Fry M. J. \& Dale T. C., Interaction of axin and Dvl-2 proteins regulates Dvl-2-stimulated TCF-dependent transcription. EMBO J., 1999, 18, 2823-2835.

Sokol S., Christian J. L., Moon R. T. \& Melton D. A., Injected Wnt RNA induces a complete body axis in Xenopus embryos. Cell, 1991, 67, 741-752.

Strutt D. I., Asymmetric localization of frizzled and the establishment of cell polarity in the Drosophila wing. Mol. Cell., 2001, 7, 367-375.

Strutt D. I., The asymmetric subcellular localisation of components of the planar polarity pathway. Semin. Cell. Dev. Biol., 2002, 13, 225-231.

Strutt D. I., Frizzled signalling and cell polarisation in Drosophila and vertebrates. Development. 2003, 130, 4501-4513.

Sussman D. J., Klingensmith J., Salinas P., Adams P. S., Nusse R. \& Perrimon N., Isolation and characterization of a mouse homolog of the Drosophila segment polarity gene dishevelled. Dev. Biol., 1994, 166, 73-86.

Tada M., Smith J. C., Xwnt11 is a target of Xenopus Brachyury: regulation of gastrulation movements via Dishevelled, but not through the canonical Wnt pathway. Development, 2000, 127, 2227-2238.

Tsang M., Lijam N., Yang Y., Beier D. R., Wynshaw-Boris A. \& Sussman D. J., Isolation and characterization of mouse dishevelled-3. Dev. Dyn., 1996, 207, 253-262.

Veeman M. T., Slusarski D. C., Kaykas A., Louie S. H. \& Moon R. T., Zebrafish prickle, a modulator of noncanonical Wnt $/ \mathrm{Fz}$ signaling, regulates gastrulation movements. Curr. Biol., 2003, 13, 680-685.

Wang J., Mark S., Zhang X., Qian D., Yoo S. J., Radde-Gallwitz K., Zhang Y., Lin X., Collazo A., Wynshaw-Boris A. \& Chen P., Regulation of polarized extension and planar cell polarity in the cochlea by the vertebrate PCP pathway. Nat. Genet., 2005, 37, 980-985.

Wang Y., Guo N. \& Nathans J., The role of Frizzled3 and Frizzled6 in neural tube closure and in the planar polarity of inner-ear sensory hair cells. J. Neurosci., 2006, 26, 21472156.

Yamamoto H., Kishida S., Kishida M., Ikeda S., Takada S. \& Kikuchi A., Phosphorylation of axin, a Wnt signal negative regulator, by glycogen synthase kinase- $3 \beta$ regulates its stability. J. Biol. Chem., 1999, 274, 10681-10684.

Yoshida N. \& Liberman M. C., Stereociliary anomaly in the guinea pig: effects of hair bundle. Hear Res., 1999, 131, 29-38.

Yost C., Torres M., Miller J. R., Huang E., Kimelman D., Moon R. T., The axis-inducing activity, stability, and subcellular distribution of $\beta$-catenin is regulated in Xenopus embryos by glycogen synthase kinase 3. Genes Dev., 1996, 10, 14431454. 